\title{
Certain Subclasses of Analysic P-Valent Functions With Respect To Other Points
}

${ }^{1}$ Hamzat J. O, ${ }^{2}$ Sangoniyi S. O.

${ }^{I}$ Department of Pure and Applied Mathematics, Ladoke Akintola University, of Technology, Ogbomoso P.M.B. 4000, Ogbomoso, Oyo State, Nigeria.

${ }^{2}$ Department of Mathematics,Emmanuel Alayande College of Education, OyoP.M.B. 1010, Oyo State, Nigeria.

Abstract: Let $T(\omega)$ be the class of analytic functions of the form:

$$
f(z)=(z-\omega)+\sum_{k=2}^{\infty} a_{k}(z-\omega)^{k}
$$

defined in the open unit disk $D=\{z:|z|<1\}$ normalized with $f(\omega)=0$ and $f^{\prime}(\omega)-1=0$ where $\omega$ is an arbitrary fixed point in $D$. The authors study certain subclasses $S_{s, n}^{*}(\omega, \alpha, \gamma, \lambda, p, l), S_{c, n}^{*}(\omega, \alpha, \gamma, \lambda, p, l)$ and $S_{s c, n}^{*}(\omega, \alpha, \gamma, \lambda, p, l) \quad$ of analytic $p$-valent function with negative coefficients in the unit disk. The results presented in this paper include coefficient bounds and distortion properties for functions belonging to these subclasses. Further results include linear combination of several functions and radii of starlikeness and convexity for functions belonging to the aforemention subclasses.

Mathematics Subject Classification: Primary 30C45

Keywords and phrases: Analytic function, Univalent function, Starlikeness, Convexity, Coefficient inequalities.

\section{Introduction}

Let $T(\omega)$ denote the class of functions of the form

$$
f(z)=(z-\omega)+\sum_{k=2}^{\infty} a_{k}(z-\omega)^{k}
$$

which are analytic and univalent in the open unit disk $D=\{z:|z|<1\}$ and normalized with $f(\omega)=0$ and $f^{\prime}(\omega)-1=0$, where $\omega$ is an arbitrary fixed point in $D . S(\omega) \subset T(\omega)$ denotes the class of analytic and univalent functions (see[1, 7,8$]$ ).

Also, let $T_{p}(\omega)$ be the class of analytic p-valent functions $f(z)$ of the form

$$
f(z)=(z-\omega)^{p}-\sum_{k=1}^{\infty} a_{p+k}(z-\omega)^{p+k} .
$$

Now, supposing we pose index alpha on (2) such hat

$$
f(z)^{\alpha}=\left[(z-\omega)^{p}-\sum_{k=1}^{\infty} a_{p+k}(z-\omega)^{p+k}\right]^{\alpha} .
$$

This is,

$$
f(z)^{\alpha}=\left[(z-\omega)^{p}-\left(a_{p+1}(z-\omega)^{p+1}+a_{p+2}(z-\omega)^{p+2}+\ldots\right)\right]^{\alpha}
$$

Expanding (3) binomially, we have

$$
f(z)^{\alpha}=(z-\omega)^{\alpha p}\left[1-\sum_{j=1}^{\infty} \alpha_{j}\left(a_{p+1}(z-\omega)+a_{p+2}(z-\omega)^{2}+\ldots\right)^{j}\right]
$$

where $\alpha_{1}=\alpha, \alpha_{2}=\frac{\alpha(\alpha-1)}{2 !}, .$.

Finally, we have 


$$
f(z)^{\alpha}=(z-\omega)^{\alpha p}-\sum_{k=1}^{\infty} a_{p+k}(z-\omega)^{\alpha p+k}
$$

Our motivation for this work is that several authors and researchers (see[1,2,3,4,5,6,7,8,9,10]) to mention but few have worked extensively on coefficient bounds for functions of the type (2), hardly will you find coefficient inequalities for the functions of the type (4) perhaps due to the difficulties the index $\alpha$ always pose. However, in the present paper, the authors aim at generalizing $\alpha(i . e . \alpha>0)$. Here, we let $T_{p}(\omega, \alpha)$ be the class of functions of the form (4) where $\alpha$ is real.

Using Aouf et al derivative operator (see[2,3]), we can write for function

$$
f(z)^{\alpha} \in T_{p}(\omega, \alpha) \text { that }
$$

$$
I_{\omega, p}^{n}(\lambda, l) f(z)^{\alpha}=\left(\frac{1+\lambda(\alpha p-1)+l}{1+l}\right)^{n}(z-\omega)^{\alpha \rho}-\sum_{k=1}^{\infty}\left(\frac{1+\lambda(\alpha p+k-1)+l}{1+l}\right)^{n} a_{P+k}(z-\omega)^{\alpha p+k}
$$

$n \in N_{0}, p \in N, l \geq 0, \lambda \geq 0, \alpha>0$ and $k \geq 1$.

At this junction, the authors wish to define the following:

\section{Definition A:}

(i.) Let the function $f(z)$ be defined by (2). Then $f(z) \in S_{n}^{*}(\omega, \lambda, p, l)$ if and only if

$$
\mathfrak{R}\left\{\frac{(z-\omega)\left(I_{\omega, p}^{n}(\lambda, l) f(z)\right)^{\prime}}{I_{\omega, p}^{n}(\lambda, l) f(z)}\right\}>0, n \in N_{0,} \quad z \in D
$$

and $S_{n}^{*}(w, \lambda, p, l)$ denote the class of $\omega-n-$ starlike functions.

(ii.) Let function $f(z)$ be defined by (2). Then $f(z) \in S_{s, n}^{*}(\omega, \lambda, p, l)$ if and only if

$$
\mathfrak{R}\left\{\frac{(z-\omega)\left(I_{\omega, p}^{n}(\lambda, l) f(z)\right)^{\prime}}{I_{\omega, p}^{n}(\lambda, l) f(z)-I_{\omega, p}^{n}(\lambda, l) f(-z)}\right\}>0, n \in N_{0}, \quad z \in D .
$$

and $S_{s, n}^{*}(\omega, \lambda, p, l)$ denotes the class of starlike functions with respect to symmetric points and $\omega$ is an arbitrary fixed point in $D$.

(iii) Let function $f(z)$ be defined by (2). Then $f(z) \in S_{s, n}^{*}(\omega, \gamma, \beta, \lambda, \rho, \iota)$ if and only if

$$
\left|\frac{(z-\omega)\left(I_{\omega, p}^{n}(\lambda, l) f(z)\right)^{\prime}}{I_{\omega, p}^{n}(\lambda, l) f(z)-I_{\omega, p}^{n}(\lambda, l) f(-z)}-p\right|<\gamma\left|\frac{\beta(z-\omega)\left(I_{\omega, p}^{n}(\lambda, l) f(z)\right)^{\prime}}{I_{\omega, p}^{n}(\lambda, l) f(z)-I_{\omega, p}^{n}(\lambda, l) f(-z)}+p\right|
$$

for some $0 \leq \beta \leq 1, \quad 0<\gamma \leq 1$ and $\mathrm{z} \in D$ where $\omega$ is an arbitrary fixed point in $D$. We

denote the class of $\omega-n-$ starlike with respect to symmetric points by $S_{s, n}^{*}(\omega, \gamma, \beta, \lambda . p . l)$.

Definition B: Let the function $f(z)^{\alpha}$ be defined by (4). Then $f(z)^{\alpha}$ is said to be $\omega$-n-starlike with respect to symmetric point if it satisfies the condition:

$$
\left|\frac{(z-\omega)\left(I_{\omega, p}^{n}(\lambda, l) f(z)^{\alpha}\right)^{\prime}}{I_{\omega, p}^{n}(\lambda, l) f(z)^{\alpha}-I_{\omega, p}^{n}(\lambda, l) f(-z)^{\alpha}}-\alpha p\right|<\gamma\left|\frac{\beta(z-\omega)\left(I_{\omega, p}^{n}(\lambda, l) f(z)^{\alpha}\right)^{\prime}}{I_{\omega, p}^{n}(\lambda, l) f(z)^{\alpha}-I_{\omega, p}^{n}(\lambda, l) f(-z)^{\alpha}}+\alpha p\right|(9)
$$

where $n \in N o=\{0\} \bigcup \mathrm{N}, 0 \leq \beta \leq 1,0<\gamma \leq 1,0 \leq \frac{\alpha p(1-\gamma)}{1+\gamma \beta}<1$ and $z \in D$. We denote the class of $\omega-n-$ starlike with respect to symmetric points by $S_{s, n}^{*}(\omega, \alpha, \gamma, \beta, \lambda, p, l)$ and $\omega$ is an arbitrary fixed point in $D$.

Definition C: Let the function $f(z)^{\alpha}$ be defined by (4). Then $f(z)^{\alpha}$ is said to be $\omega-n-$ starlike with respect to conjugate point if it satisfies the condition: 


$$
\left|\frac{(z-\omega)\left(I_{w, p}^{n}(\lambda, l) f(z)^{\alpha}\right)}{I_{\omega, p}^{n}(\lambda, l) f(z)^{\alpha}+\overline{I_{\omega, p}^{n}(\lambda, l) f(\bar{z})^{\alpha}}}-\alpha p\right|<\gamma\left|\frac{\beta(z-\omega)\left(I_{w, p}^{n}(\lambda, l) f(z)^{\alpha}\right)}{I_{\omega, p}^{n}(\lambda, l) f(z)^{\alpha}+\overline{I_{\omega, p}^{n}(\lambda, l) f(\bar{z})^{\alpha}}}+\alpha p\right|
$$

where $n \in N_{0}=\{0\} \cup N, 0 \leq \beta \leq 1,0<\gamma \leq 1,0 \leq \frac{\alpha p(1-\gamma)}{1+\gamma \beta}<1, z \in D$ and $\omega$ is arbitrarily fixed in D. We denote the class of $\omega-n-$ starlike with respect to symmetric points by $S_{c . n}^{*}(\omega, \alpha, \gamma, \beta, \lambda, p, l)$.

Definition D: Let the functions $f(z)^{\alpha}$ be defined by (4). Then $f(z)^{\alpha}$ is said to be $\omega-n$-starlike with respect to symmetric conjugate points if satisfies the condition:

$$
\left|\frac{(z-\omega)\left(I_{w, p}^{n}(\lambda, l) f(z)^{\alpha}\right)}{I_{\omega, p}^{n}(\lambda, l) f(z)^{\alpha}-\overline{I_{\omega, p}^{n}(\lambda, l) f(\overline{-z})^{\alpha}}}-\alpha p\right|<\gamma\left|\frac{\beta(z-\omega)\left(I_{w, p}^{n}(\lambda, l) f(z)^{\alpha}\right)^{\prime}}{I_{\omega, p}^{n}(\lambda, l) f(z)^{\alpha}-\overline{I_{\omega, p}^{n}(\lambda, l) f(\overline{-z})^{\alpha}}}+\alpha p\right|
$$

where $n \in N_{0}=\{0\} \cup N, 0 \leq \beta \leq 1,0<\gamma \leq 1,0 \leq \frac{\alpha p(1-\gamma)}{1+\gamma \beta}<1, z \in D$ and $\omega$ is arbitrarily fixed in $D$. We denote the class of $\omega$-n-starlike with respect to symmetric points by $S_{s c, n}^{*}(\omega, \alpha, \gamma, \beta, \lambda, p, l)$.

\section{Coefficient Inequalities}

Theorem 2.1: Let the function $f(z)^{\alpha}$ be defined by (4) and $I_{\omega, p}^{n}(\lambda, l) f(z)^{\alpha}-I_{\omega, p}^{n}(\lambda, l) f(-z)^{\alpha} \neq 0$ for $z \in \omega$. Then, $f(z)^{\alpha} \in S_{s, n}^{*}(\omega, \alpha, \gamma, \beta, \lambda, \rho, l)$ if and only if

$$
\begin{gathered}
\sum_{k=1}^{\infty}(r+d)^{k}\left[k(1+\gamma \beta)+\alpha p\left(\gamma\left(\beta+1-(-1)^{\alpha p}\right)+(-1)^{\alpha p+\kappa}\right)\right]\left(\frac{1+\lambda(\alpha p+k-1+l)}{1+l}\right)^{n} a_{p+k}(\alpha) \\
\leq \alpha p\left[\gamma\left(\beta+1-(-1)^{\alpha p}\right)+(-1)^{\alpha p}\right]\left(\frac{1+\lambda(\alpha p-1)+l}{1+l}\right)^{n}
\end{gathered}
$$

Proof: using (4), (5) and (9), that is

$$
\begin{gathered}
\left|\frac{(z-\omega)\left(I_{\omega, p}^{n}(\lambda, l) f(z)^{\alpha}\right)}{I_{\omega, p}^{n}(\lambda, l) f(z)^{\alpha}-I_{\omega, p}^{n}(\lambda, l) f(-z)^{\alpha}}-\alpha p\right|<\gamma \mid \frac{\beta(z-\omega)\left(I_{\omega, p}^{n}(\lambda, l) f(z)^{\alpha}\right)}{I_{\omega, p}^{n}(\lambda, l) f(z)^{\alpha}-I_{\omega, p}^{n}(\lambda, l) f(-z)^{\alpha}}+\alpha p \\
f(z)^{\alpha}=(z-\omega)^{\alpha \rho}-\sum_{k=1}^{\infty} a p+k(\alpha)(z-\omega)^{\alpha \rho^{+k}}
\end{gathered}
$$

and

$$
\begin{gathered}
I_{\omega, p}^{n}(\lambda, l) f(z)^{\alpha} \\
=\left(\frac{1+\lambda(\alpha p-1)+l}{1+l}\right)^{n}(z-\omega)^{\alpha p}-\sum_{k=1}^{\infty}\left(\frac{1+\lambda(\alpha p+k-1)+l}{1+l}\right)^{n} \alpha_{p+k}(z-\omega)^{\alpha p+k} .
\end{gathered}
$$

For convenience, we let

$$
f(-z)^{\alpha}=(-1)^{\alpha p}(z-\omega)^{\alpha p}-\sum_{k=1}^{\infty}(-1)^{\alpha p+k} a_{p+k}(\alpha)(z-\omega)^{\alpha p+k}
$$

and

$$
I_{\omega, p}^{n}(\lambda, l) f(-z)^{\alpha}
$$


$=(-1)^{\alpha p}\left(\frac{1+\lambda(\alpha p-1)+l}{1+l}\right)^{n}(z-\omega)^{\alpha p}-\sum_{k=1}^{\infty}(-1)^{\alpha p+k}\left(\frac{1+\lambda(\alpha p+k-1)+l}{1+l}\right)^{n} a_{p+k}(\alpha)(z-\omega)^{\alpha p+k}$

This implies that

$$
\begin{aligned}
& \mid \alpha p(-1)^{\alpha p}\left(\frac{1+\lambda(\alpha p-1)+l}{1+l}\right)^{n}(z-\omega)^{\alpha p}-\sum_{k=1}^{\infty}\left[\alpha p(-1)^{\alpha p+k}+k\right]\left(\frac{1+\lambda(\alpha p+k-1)+l}{1+l}\right)^{n} a_{p+k}(\alpha)(z-\omega)^{\alpha p+k} \mid \\
&<\gamma \mid \alpha p\left(\beta+1-(-1)^{\alpha p}\right)\left(\frac{1+\lambda(\alpha p-1)+l}{1+l}\right)^{n} \\
& \quad-\sum_{k=1}^{\infty}\left[k \beta+\alpha p\left(\beta+1-(-1)^{\alpha p}\right)\right]\left(\frac{1+\lambda(\alpha p+k-1)+l}{1+l}\right)^{n} a_{p+k}(\alpha)(z-\omega)^{\alpha p+k} \mid .
\end{aligned}
$$

That is

$$
\begin{gathered}
-\alpha p\left[(-1)^{\alpha p}+\gamma\left(\beta+1-(-1)^{\alpha p}\right)\right]\left(\frac{1+\lambda(\alpha p-1)+l}{1+l}\right)^{n}|z-\omega|^{\alpha p} \\
+\sum_{k=1}^{\infty}\left[k+\alpha p(-1)^{\alpha p+k}+\gamma\left(k \beta+\alpha p\left(\beta+1-(-1)^{\alpha p+k}\right)\right]\left(\frac{1+\lambda(\alpha p+k-1)+l}{1+l}\right)^{n} a_{p+k}(\alpha)|z-\omega|^{\alpha p+k} \leq 0 .\right.
\end{gathered}
$$

Letting $|z-\omega|=r+d$, we have

$$
\begin{aligned}
\sum_{k=1}^{\infty}[k+ & \left.\alpha p(-1)^{\alpha p+k}+\gamma\left(k \beta+\alpha p\left(\beta+1-(-1)^{\alpha p+k}\right)\right)\left(\frac{1+\lambda(\alpha p+k-1)+l}{1+l}\right)^{n} a_{p+k}(\alpha)(r+d)^{\alpha p+k}\right] \\
& -\alpha p\left[\gamma\left(\beta+1-(-1)^{\alpha p}\right)+(-1)^{\alpha p}\right]\left(\frac{1+\lambda(\alpha p-1)+l}{1+l}\right)^{n} \leq 0 .
\end{aligned}
$$

Therefore, by the maximum modulus theorem, we have $f(z)^{\alpha} \in S_{s, n}^{*}(\omega, \alpha, \gamma, \beta, \lambda, p, l)$.

For the converse, let us suppose that

This implies that,

$$
\left|\frac{\frac{(z-\omega)\left(I_{\omega, p}^{n}(\lambda, l) f(z)^{\alpha}\right)}{I_{\omega, p}^{n}(\lambda, l) f(z)^{\alpha}-I_{\omega, p}^{n}(\lambda, l) f(-z)^{\alpha}}-\alpha \rho}{\frac{\beta(z-\omega)\left(\mathrm{I}_{\omega, p}^{n}(\lambda, l) f(z)^{\alpha}\right)}{\mathrm{I}_{\omega, p}^{n}(\lambda, l) f(z)^{\alpha}-I_{\omega, p}^{n}(\lambda, l) f(-z)^{\alpha}}+\alpha \rho}\right|<\gamma .
$$

$\left|\frac{-\left[-\alpha p(-1)^{\alpha p} \varsigma(z-\omega)^{\alpha p}+\sum_{k=1}^{\infty}\left[k+\alpha p(-1)^{\alpha p+k}\right] \eta a_{p+k}(\alpha)(z-\omega)^{\alpha p+\kappa}\right]}{\alpha p\left[\beta+1-(-1)^{\alpha p}\right] \varsigma(z-\omega)^{\alpha p}-\sum_{k=1}^{\infty}\left[k \beta+\alpha p\left(\beta+1-(-1)^{\alpha p+k}\right)\right] \eta a_{p+k}(\alpha)(z-\omega)^{\alpha p+k}}\right|<\gamma$

where

$$
\varsigma=\left(\frac{1+\lambda(\alpha p-1)+l}{1+l}\right)^{n} \text { and } \eta=\left(\frac{1+\lambda(\alpha p+k-1)+l}{1+l}\right)^{n} .
$$

Since $\mathfrak{R}(z-\omega) \leq|z-\omega|$ for all $z$, then we have

$$
\mathfrak{R}\left\{\frac{-\alpha p(-1)^{\alpha p} \varsigma+\sum_{k=1}^{\infty}\left[k+\alpha p(-1)^{\alpha \rho+k}\right] \eta a_{p+k}(\alpha)(r+d)^{k}}{\alpha p\left[\beta+1-(-1)^{\alpha p}\right] \varsigma-\sum_{k=1}^{\infty}\left[k \beta+\alpha p\left(\beta+1-(-1)^{\alpha p+k}\right)\right] \eta a_{p+k}(\alpha)(r+d)^{k}}\right\}<\gamma
$$


If we choose values of $\mathrm{z}$ on the real axis so that $\frac{(z-\omega)\left(I_{w, p}^{n}(\lambda, l) f(z)^{\alpha}\right)^{j}}{I_{\omega, p}^{n}(\lambda, l) f(z)^{\alpha}-I_{\omega, p}^{n}(\lambda, l) f(-z)^{\alpha}}$ is real, $I_{\omega, p}^{n}(\lambda, l) f(z)^{\alpha}-I_{\omega, p}^{n}(\lambda, l) f(-z)^{\alpha} \neq 0$, for $z \neq \omega$ and upon clearing the denominator in (13). Also letting $|z-\omega| \rightarrow(r+d)$ through the real values, then we obtain

$$
\begin{gathered}
\sum_{k=1}^{\infty}\left(k+\alpha p(-1)^{\alpha p+k}\right) \eta a_{p+k}(\alpha)(r+d)^{k}+\sum_{k=1}^{\infty} \gamma\left[k \beta+\alpha p\left(\beta+1-(-1)^{\alpha p+k}\right)\right] \eta a_{p+k}(\alpha)(r+d)^{k} \\
\leq \alpha p \gamma\left(\beta+1-(-1)^{\alpha p}\right) \zeta+\alpha p(-1)^{\alpha p} \varsigma
\end{gathered}
$$

and this completes the proof of theorem 2.1.

Corollary 2.1: Let the function $f(z)^{\alpha}$ defined by (4) be in the class $S_{s, n}^{*}(\omega, \alpha, \gamma, \beta, \lambda, p, l)$.

Then, we have

$$
a_{p+k} \leq \frac{\alpha p\left[\gamma\left(\beta+1-(-1)^{\alpha \rho}\right)+(-1)^{\alpha p}\right] \varsigma}{\left[k+\alpha p(-1)^{\alpha p+k}+\gamma\left(k \beta+\alpha p\left(\beta+1-(-1)^{\alpha p+k}\right)\right)\right] \eta(r+d)^{k}} \quad k \geq 1, n \in N_{0} \text { and } z \in D
$$

where $\varsigma$ and $\eta$ are as earlier defined.

The equality in (14) is attained for function $f(z)^{\alpha}$ given by

$$
f(z)^{\alpha}=(z-\omega)^{\alpha p}-\frac{\alpha p\left[\gamma\left(\beta+1-(-1)^{\alpha p}\right)+(-1)^{\alpha p}\right] \varsigma}{\left[k+\alpha p(-1)^{\alpha p}+\gamma\left(k \beta+\alpha p\left(\beta+1-(-1)^{\alpha p+k}\right)\right)\right] \eta(r+d)^{k}}(z-\omega)^{\alpha p+k} .
$$

Theorem 2.2: Let the function $f(z)^{\alpha}$ be defined by (4). Then $f(z)^{\alpha} \in S_{c, n}^{*}(\omega, \alpha, \gamma, \beta, \lambda, p, l)$ if and only if

$$
\sum_{k=1}^{\infty}[k(1+\gamma \beta)+\alpha p(\gamma(\beta+2)-1)] \eta a_{p+k}(\alpha)(r+d)^{k} \leq \alpha p[\gamma(\beta+2)-1] \varsigma
$$

Proof: Using (4), (5) and (10), then

$$
\begin{gathered}
\left|\frac{(z-\omega)\left(I_{w, p}^{n}(\lambda, l) f(z)^{\alpha}\right)}{I_{\omega, p}^{n}(\lambda, l) f(z)^{\alpha}+\overline{I_{\omega, p}^{n}(\lambda, l) f(\bar{z})^{\alpha}}}-\alpha p\right|<\gamma \mid \frac{\beta(z-\omega)\left(I_{w, p}^{n}(\lambda, l) f(z)^{\alpha}\right)}{\mid I_{\omega, p}^{n}(\lambda, l) f(z)^{\alpha}+I_{\omega, p}^{n}(\lambda, l) f(\bar{z})^{\alpha}}+\alpha p \\
f(z)^{\alpha}=(z-\omega)^{\alpha p}-\sum_{k=1}^{\infty} a_{p+k}(\alpha)(z-\omega)^{\alpha p+k}
\end{gathered}
$$

and

$$
\begin{gathered}
I_{\omega, p}^{n}(\lambda, l) f(z)^{\alpha} \\
=\left(\frac{1+\lambda(\alpha p-1)+l}{1+l}\right)^{n}(z-\omega)^{\alpha p}-\sum_{k=1}^{\infty}\left(\frac{1+\lambda(\alpha p+k-1)+l}{1+l}\right)^{n} a_{p+k}(z-\omega)^{\alpha p+k}
\end{gathered}
$$

Let

and

$$
f(\bar{z})^{\alpha}=(z-\omega)^{\alpha p}+\sum_{k=1}^{\infty} a_{p+k}(\alpha)(z-\omega)^{\alpha p+k}
$$

$$
\begin{gathered}
\overline{I_{\omega, p}^{n}(\lambda, l) f(\bar{z})^{\alpha}} \\
=\left(\frac{1+\lambda(\alpha p-1)+l}{1+l}\right)^{n}(z-\omega)^{\alpha p}-\sum_{k=1}^{\infty}\left(\frac{1+\lambda(\alpha p+k-l)}{1+l}\right)^{n} a_{p+k}(\alpha)(z-\omega)^{\alpha p+k} .
\end{gathered}
$$


From (10), we have

$$
\begin{aligned}
& \left|-\alpha p\left(\frac{1+\lambda(\alpha p-1)+l}{1+l}\right)^{n}(z-\omega)^{\alpha p}-\sum_{k=1}^{\infty}(k-\alpha p)\left(\frac{1+\lambda(\alpha p+k-1)+l}{1+l}\right)^{n} a_{p+k}(\alpha)(z-\omega)^{\alpha p+k}\right| \\
& <\mid \alpha p \gamma(\beta+2)\left(\frac{1+\lambda(\alpha p-1)+l}{1+l}\right)^{n}(z-\omega)^{\alpha p} \\
& \quad-\sum_{k=1}^{\infty} \gamma(\beta(k+\alpha p)+2 \alpha p)\left(\frac{1+\lambda(\alpha p+k-1)+l}{1+l}\right)^{n} a_{p+k}(\alpha)(z-\omega)^{\alpha p+k} \mid .
\end{aligned}
$$

This implies that

$$
\begin{aligned}
& \alpha p(1-\gamma(\beta+2))\left(\frac{1+\lambda(\alpha p-1)+l}{1+l}-\right)^{n}|z-\omega|^{\alpha p} \\
& +\sum_{k=1}^{\infty}[k+\alpha p(2 \gamma-1)+\beta \gamma(k+\alpha p)]\left(\frac{1+\lambda(\alpha p+k-1)+l}{1+l}\right)^{n} a_{p+k}(\alpha)|z-\omega|^{\alpha p+k} \geq 0 .
\end{aligned}
$$

Hence,

$$
\begin{gathered}
\sum_{k=1}^{\infty}[k+\alpha p(2 \gamma-1)+\beta \gamma(k+\alpha p)]\left(\frac{1+\lambda(\alpha p+k-1)+l}{1+l}\right)^{n} a_{p+k}(\alpha)|z-\omega|^{\alpha p+k} \\
\leq \alpha p[\gamma(\beta+2)-1]\left(\frac{1+\lambda(\alpha p-1)+l}{1+l}\right)^{n}
\end{gathered}
$$

Letting $|z-\omega|=r+d$, we obtain

$$
\begin{gathered}
\sum_{k=1}^{\infty \infty \infty}[k+\alpha p(2 \gamma-1)+\beta \gamma(k+\alpha p)]\left(\frac{1+\lambda(\alpha p+k-1)+l}{1+l}\right)^{n} a_{p+k}(\alpha)(r+d)^{k} \\
\leq \alpha p[\gamma(\beta+2)-1]\left(\frac{1+\lambda(\alpha p-1)+l}{1+l}\right)^{n}
\end{gathered}
$$

and the complete the proof of Theorem 2.2.

Corollary 2.2: Let the function $f(z)^{\alpha}$ defined by (4) be in the class $S_{c, n}^{*}(\omega, \alpha, \gamma, \beta, \lambda, p, l)$.

Then, we have

$$
a_{p+k}(\alpha) \leq \frac{\alpha p[\gamma(\beta+2)-1]\left(\frac{1+\lambda(\alpha p-1)+l}{1+l}\right)^{n}}{[k(1+\gamma \beta)+\alpha p(\gamma(\beta+2)-1)]\left(\frac{1+\lambda(\alpha \rho+k-1)+l}{1+l}\right)^{n} a_{p+k}(\alpha)(r+d)^{k}}
$$

The equality in (17) is attained for the function $f(z)^{\alpha}$ given by

$$
\begin{aligned}
& f(z)^{\alpha}=(z-\omega)^{\alpha p}-\frac{\alpha p[\gamma(\beta+2)-1]\left(\frac{1+\lambda(\alpha p-1)+l}{1+l}\right)^{n}}{[k(1+\gamma \beta)+\alpha p(\gamma(\beta+2)-1)]\left(\frac{1+\lambda(\alpha p-1)+l}{1+l}\right)^{n} a_{p+k}(\alpha)(r+d)^{k}}(z-\omega)^{\alpha p+k} \\
& k \geq 1, n \in N o \text { and } \mathrm{z} \in D .
\end{aligned}
$$

Theorem 2.3: Let the function $f(z)^{\alpha}$ defined by (4) be in the class $S_{s c, n}^{*}(\omega, \alpha, \gamma, \beta, \lambda, p, l)$.

Then, we have 


$$
\begin{aligned}
& \sum_{k=1}^{\infty}\left[k(1+\gamma \beta)+\alpha p\left(\gamma(1+\beta)+(1-\gamma)(-1)^{\alpha p+k}\right)\right]\left(\frac{1+\lambda(\alpha p+k-1)+l}{1+l}\right)^{n} a_{p+k}(\alpha)(r+d)^{k} \\
& \quad \leq \alpha p\left[\gamma\left(\beta+1-(-1)^{\alpha p}\right)+(-1)^{\alpha p}\right]\left(\frac{1+\lambda(\alpha p-1)+l}{1+l}\right)^{n}
\end{aligned}
$$

Proof 2.3: Using (4),(5) and (11), we have

$$
\left|\frac{(z-\omega)\left(I_{w, p}^{n}(\lambda, l) f(z)^{\alpha}\right)}{I_{\omega, p}^{n}(\lambda, l) f(z)^{\alpha}-\overline{I_{\omega, p}^{n}(\lambda, l) f(\overline{-z})^{\alpha}}}-\alpha p\right|<\gamma\left|\frac{\beta(z-\omega)\left(I_{w, p}^{n}(\lambda, l) f(z)^{\alpha}\right)^{\prime}}{I_{\omega, p}^{n}(\lambda, l) f(z)^{\alpha}-\overline{I_{\omega, p}^{n}(\lambda, l) f(\overline{-z})^{\alpha}}}+\alpha p\right|
$$

and

$$
\begin{gathered}
\overline{I_{\omega, p}^{n}(\lambda, p) f(\overline{-z})^{\alpha}} \\
=(-1)^{\alpha p}\left(\frac{1+\lambda(\alpha p-1)+l}{1+l}\right)^{n}(z-\omega)^{\alpha p}-\sum_{k=1}^{\infty}(-1)^{\alpha p+k}\left(\frac{1+\lambda(\alpha p+k-1)+l}{1+l}\right)^{n} a_{p+k}(\alpha)(z-\omega)^{\alpha p+k}
\end{gathered}
$$

From (11),we have

$$
\begin{aligned}
& \mid-\left[-\alpha p(-1)^{\alpha p}\left(\frac{1+\lambda(\alpha p-1)+l}{1+l}\right)^{n}(z-\omega)^{\alpha p}\right. \\
& \left.+\sum_{k=1}^{\infty}\left[k+\alpha p(-1)^{\alpha p+k}\right]\left(\frac{1+\lambda(\alpha p+k-1)+l}{1+l}\right)^{n} a_{p+k}(\alpha)(z-\omega)^{\alpha p+k}\right] \mid \\
& \quad-\gamma \mid \alpha p\left(\beta+1-(-1)^{\alpha p}\right)\left(\frac{1+\lambda(\alpha p-1)+l}{1+l}\right)^{n}(z-\omega)^{\alpha p} \\
& -\sum_{k=1}^{\infty}\left[\beta(\alpha p+k)+\alpha p\left(1-(-1)^{\alpha p+k}\right)\right]\left(\frac{1+\lambda(\alpha p+k-1)+l}{1+l}\right)^{n} a_{p+k}(\alpha)(z-\omega)^{\alpha p+k} \mid<0 .
\end{aligned}
$$

This implies that

$$
\begin{gathered}
\sum_{k=1}^{\infty}\left[k+\gamma(\alpha p(1+\beta)+\beta k)+\alpha p(1-\gamma)(-1)^{\alpha p+k}\right]\left(\frac{1+\lambda(\alpha p+k-1)+l}{1+l}\right)^{n} a_{p+k}(\alpha)|z-\omega|^{\alpha p+k} \\
-\alpha p\left[\gamma\left(\beta+1-(-1)^{\alpha p}\right)+(-1)^{\alpha p}\right]\left(\frac{1+\lambda(\alpha p-1)+l}{1+l}\right)^{n}|z-\omega|^{\alpha p} \leq 0 .
\end{gathered}
$$

Letting $|z-\omega|=r+d$, then we have

$$
\begin{gathered}
\sum_{k=1}^{\infty}\left[k(1+\gamma \beta)+\alpha p\left(\gamma(1+\beta)+(1-\gamma)(-1)^{\alpha p+k}\right)\right]\left(\frac{1+\lambda(\alpha p+k-1)+l}{1+l}\right)^{n} a_{p+k}(\alpha)(r+d)^{k} \\
\leq \alpha p\left[\gamma\left(\beta+1-(-1)^{\alpha p}\right)+(-1)^{\alpha p}\right]\left(\frac{1+\lambda(\alpha p-1)+l}{1+l}\right)^{n} .
\end{gathered}
$$

and this ends the proof of theorem 2.3.

Corollary2.3: Let the function $f(z)^{\alpha}$ defined by (4) be in the class $S_{s c, n}^{*}(\omega, \alpha, \gamma, \beta, \lambda, p, l)$. Then, we have 


$$
a_{p+k}(\alpha) \leq \frac{\alpha p\left[\gamma\left(\beta+1-(-1)^{\alpha p}\right)+(-1)^{\alpha p}\right]\left(\frac{1+\lambda(\alpha p-1)+l}{1+l}\right)^{n}}{\left[k+\gamma(\alpha p(1+\beta)+\beta k)+\alpha p(1-\gamma)(-1)^{\alpha p+k}\right]\left(\frac{1+\lambda(\alpha p+k-1)+l}{1+l}\right)^{n} a_{p+k}(\alpha)(r+d)^{k}}
$$

$$
k \geq 1 \text { and } n \in N_{o} .
$$

The equality in (20) is attained for the function $f(z)^{\alpha}$ given by

$$
\begin{gathered}
f(z)^{\alpha}= \\
(z-\omega)^{\alpha p}-\frac{\alpha p\left[\gamma\left(\beta+1-(-1)^{\alpha p}\right)+(-1)^{\alpha p}\right]\left(\frac{1+\lambda(\alpha p-1)+l}{1+l}\right)^{n}}{\left[k+\gamma(\alpha p(1+\beta)+\beta k)+\alpha p(1-\gamma)(-1)^{\alpha p+k}\right]\left(\frac{1+(\alpha p+k-1)+l}{1+l}\right)^{n} a_{p+k}(\alpha)(r+d)^{k}}(z-\omega)^{\alpha p+k} \\
k \geq 1 \text { and } n \in N_{o}
\end{gathered}
$$

\section{Distortion Theorem}

Theorem 3.1: Let the function $f(z)^{\alpha}$ be defined by (4) be in the class $S_{s, n}^{*}(\omega, \alpha, \gamma, \beta, \lambda, p, l)$.

Then, we have

$$
\begin{gathered}
\begin{aligned}
&(r+d)^{\alpha p}\left(\frac{1+\lambda(\alpha p-1)+l}{1+l}\right)^{i}- \frac{\alpha p(r+d)^{\alpha p}\left[\gamma\left(\beta+1-(-1)^{\alpha p}\right)+(-1)^{\alpha p}\right]\left(\frac{1+\lambda(\alpha p-1)+l}{1+l}\right)^{n}}{\left[1+\gamma \beta+\alpha p\left(\gamma\left(\beta+1-(-1)^{\alpha p+1}\right)+(-1)^{\alpha p+1}\right)\right]\left(\frac{1+\lambda \alpha p+l}{1+l}\right)^{n-i}} \\
& \leq(r+d)^{\alpha p}\left(\frac{1+\lambda(\alpha p-1)+l}{1+l}\right)^{i}+\frac{\alpha p(r+d)^{\alpha p}\left[\gamma\left(\beta+1-(-1)^{\alpha p}\right)+(-1)^{\alpha p}\right]\left(\frac{1+\lambda(\alpha p-1)+l}{1+l}\right)^{i}}{\left[1+\gamma \beta+\alpha p\left(\gamma\left(\beta+1-(-1)^{\alpha p+1}\right)+(-1)^{\alpha p+1}\right)\right]\left(\frac{1+\lambda \alpha p+l}{1+l}\right)^{n-i}}
\end{aligned}
\end{gathered}
$$

for $z \in D$, where $0 \leq i \leq n$ and $\omega$ is arbitrarily fixed in $D$. The result is best possible.

Proof 2.3: We note that $f(z)^{\alpha} \in S_{s, n}^{*}(\omega, \alpha, \gamma, \beta, \lambda, p, l)$. if and only if $I_{\omega, p}^{i}(\lambda, l) f(z)^{\alpha} \in S_{s, n-i}^{*}(\omega, \alpha, \gamma, \beta, \lambda, p, l)$

and

$$
I_{\omega, p}^{i}(\lambda, l) f(z)^{\alpha}=\left(\frac{1+\lambda(\alpha p-1)+l}{1+l}\right)^{i}(z-\omega)^{\alpha p}-\sum_{k=1}^{\infty}\left(\frac{1+\lambda \alpha p+l}{1+l}\right)^{i} a_{p+k}(z-\omega)^{\alpha p+k} .
$$

Now, using theorem 2.1 we have

$$
\sum_{k=1}^{\infty}\left(\frac{1+\lambda(\alpha p+k-1)+l}{1+l}\right)^{i} a_{p+k}(\alpha)
$$




$$
\leq \frac{\alpha p\left[\gamma\left(\beta+1-(-1)^{\alpha p}\right)+(-1)^{\alpha p}\right]\left(\frac{1+\lambda(\alpha p-1)+l}{1+l}\right)^{n}\left(\frac{1+\lambda \alpha p+l}{1+l}\right)^{i}}{\left[1+\gamma \beta+\alpha p\left(\gamma\left(\beta+1-(-1)^{\alpha p+1}\right)+(-1)^{\alpha p+1}\right)\right]\left(\frac{1+\lambda \alpha p+l}{1+l}\right)^{n}(r+d)} .
$$

That is,

$$
\begin{gathered}
\sum_{k=1}^{\infty}\left(\frac{1+\lambda(\alpha+k-1)+l}{1+l}\right)^{i} a_{p+k}(\alpha) \\
\leq \frac{\alpha p\left[\gamma\left(\beta+1-(-1)^{\alpha p}\right)+(-1)^{\alpha p}\right]\left(\frac{1+\lambda(\alpha p-1)+l}{1+l}\right)^{n}}{\left[1+\gamma \beta+\alpha p\left(\gamma\left(\beta+1-(-1)^{\alpha p+1}\right)+(-1)^{\alpha p+1}\right)\right]\left(\frac{1+\lambda \alpha p+l}{1+l}\right)^{n-i}(r+d)} .
\end{gathered}
$$

It follow from (23) and (25) that

$$
\begin{gathered}
\quad\left|I_{\omega, p}^{i}(\lambda, l) f(z)^{\alpha}\right| \\
\geq\left(\frac{1+\lambda(\alpha p-1)+l}{1+l}\right)^{i}|z-\omega|^{\alpha p}-\sum_{k=1}^{\infty}\left(\frac{1+\lambda(\alpha p+k-1)+l}{1+l}\right)^{i} a_{p+k}(\alpha)|z-\omega|^{\alpha p+k} \\
\geq\left(\frac{1+\lambda(\alpha p-1)+l}{1+l}\right)^{i}-(r+d)^{\alpha p+1} \sum_{k=1}^{\infty}\left(\frac{1+\lambda(\alpha p+k-1)+l}{1+l}\right)^{i} a_{p+k}(\alpha) \\
\geq(r+d)^{\alpha p}\left(\frac{1+\lambda(\alpha p-1)+l}{1+l}\right)^{i}-\frac{\alpha p(r+d)^{\alpha p}\left[\gamma\left(\beta+1-(-1)^{\alpha p}\right)+(-1)^{\alpha p}\right]\left(\frac{1+\lambda(\alpha p-1)+l}{1+l}\right)^{n}}{\left[1+\gamma \beta+\alpha p\left(\gamma\left(\beta+1-(-1)^{\alpha p+1}\right)+(-1)^{\alpha p+1}\right)\right]\left(\frac{1+\lambda \alpha p+l}{1+l}\right)^{n-i}}
\end{gathered}
$$

nd also

$$
\begin{gathered}
\left|I_{\omega, p}^{i}(\lambda, l) f(z)^{\alpha}\right| \\
\leq(r+d)^{\alpha p}\left(\frac{1+\lambda(\alpha p-1)+l}{1+l}\right)^{i}+\frac{\alpha p(r+d)^{\alpha p}\left[\gamma\left(\beta+1-(-1)^{\alpha p}\right)+(-1)^{\alpha p}\right]\left(\frac{1+\lambda(\alpha p-1)+l}{1+l}\right)^{n}}{\left[1+\gamma \beta+\alpha p\left(\gamma\left(\beta+1-(-1)^{\alpha p+1}\right)+(-1)^{\alpha p+1}\right)\right]\left(\frac{1+\lambda \alpha p+l}{1+l}\right)^{n-i}}
\end{gathered}
$$

Finally, we see that the inequality in (22) is attained by the function

$$
\begin{aligned}
& I_{\omega, p}^{i}(\lambda, l) f(z)^{\alpha}=\left(\frac{1+\lambda(\alpha p-1)+l}{1+l}\right)^{i}(z-\omega)^{\alpha p} \\
& -\frac{\alpha p\left[\gamma\left(\beta+1-(-1)^{\alpha p}\right)+(-1)^{\alpha p}\right]\left(\frac{1+\lambda(\alpha p-1)+l}{1+l}\right)^{n}}{\left[1+\gamma \beta+\alpha p\left(\gamma\left(\beta+1-(-1)^{\alpha p+1}\right)+(-1)^{\alpha p+1}\right)\right]\left(\frac{1+\lambda \alpha p+l}{1+l}\right)^{n-i}(r+d)}(z-\omega)^{\alpha p+k}
\end{aligned}
$$

or by 


$$
f(z)^{\alpha}=(z-\omega)^{\alpha p}-\frac{\alpha p\left[\gamma\left(\beta+1-(-1)^{\alpha p}\right)+(-1)^{\alpha p}\right]\left(\frac{1+\lambda(\alpha p-1)+l}{1+l}\right)^{n}}{\left[1+\gamma \beta+\alpha p\left(\gamma\left(\beta+1-(-1)^{\alpha p+1}\right)+(-1)^{\alpha p+1}\right)\right]\left(\frac{1+\lambda \alpha p+l}{1+l}\right)^{n}(r+d)}(z-\omega)^{\alpha p+k}
$$

and this complete the proof of theorem 3.1.

Corollary 3.1: Let the function $f(z)^{\alpha}$ be defined by (4) be in the class $S_{s, n}^{*}(\omega, \alpha, \gamma, \beta, \lambda, \rho, l)$. Then, we have

$$
\begin{gathered}
(r+d)^{\alpha p}-\frac{\alpha p\left[\gamma\left(\beta+1-(-1)^{\alpha p}\right)+(-1)^{\alpha p}\right]\left(\frac{1+\lambda(\alpha p-1)+l}{1+l}\right)^{n}}{\left[1+\gamma \beta+\alpha p\left(\gamma(\beta+1-(-1))^{\alpha p+1}\right)+(-1)^{\alpha p+1}\right]\left(\frac{1+\lambda \alpha p+l}{1+l}\right)^{n}} \leq\left|f(z)^{\alpha}\right| \\
\leq(r+d)^{\alpha p}+\frac{\alpha p\left[\gamma\left(\beta+1-(-1)^{\alpha p}\right)+(-1)^{\alpha p}\right]\left(\frac{1+\lambda(\alpha p-1)+l}{1+l}\right)^{n}}{\left[1+\gamma \beta+\alpha p\left(\gamma(\beta+1-(-1))^{\alpha p+1}\right)+(-1)^{\alpha p+1}\right]\left(\frac{1+\lambda \alpha p+l}{1+l}\right)^{n}}
\end{gathered}
$$

for $z \in D$, where $\omega$ is arbitrarily fixed in $D$. The result is best possible for the function $f(z)^{\alpha}$ given by (29). Proof: For $i=0$ in theorem 3.1, we can easily show (30).

Theorem 3.2: Let the function $f(z)^{\alpha}$ defined by (4) be in the class $S_{c, n-i}^{*}(\omega, \alpha, \gamma, \beta, \lambda, p, l)$.

Then, we have

$$
\begin{array}{r}
(r+d)^{\alpha p}\left(\frac{1+\lambda(\alpha p-1)+l}{1+l}\right)^{l}-\frac{\alpha p(r+d)^{\alpha p}[\gamma(\beta+2)-1]\left(\frac{1+\lambda(\alpha p-1)+l}{1+l}\right)^{n}}{[1+\gamma \beta+\alpha p(\gamma(\beta+2)-1)]\left(\frac{1+\lambda \alpha \rho+l}{1+l}\right)^{n-i}} \\
\quad \leq\left|I_{\omega, p}^{i}(\lambda, l) f(z)^{\alpha}\right| \\
\leq(r+d)^{\alpha p}\left(\frac{1+\lambda(\alpha p-1)+l}{1+l}\right)^{i}+\frac{\alpha p(r+d)^{\alpha p}[\gamma(\beta+2)-1]\left(\frac{1+\lambda(\alpha p-1)+l}{1+l}\right)^{n}}{[1+\gamma \beta+\alpha p(\gamma(\beta+2)-1)]\left(\frac{1+\lambda \alpha p+l}{1+l}\right)^{n-i}}
\end{array}
$$

for $z \in D$, where $0 \leq i \leq n$ and $\omega$ is arbitrarily fixed in $D$. The result is best possible for the function $f(z)^{\alpha}$ given by

$$
\begin{gathered}
I_{\omega, p}^{i}(\lambda, l) f(z)^{\alpha} \\
=\left(\frac{1+\lambda(\alpha p-1)+l}{1+l}\right)^{i}(z-\omega)^{\alpha p}-\frac{\alpha p[\gamma(\beta+2)-1]\left(\frac{1+\lambda(\alpha p-1)+l}{1+l}\right)^{n}}{[1+\gamma \beta+\alpha p(\gamma(\beta+2)-1)]\left(\frac{1+\lambda \alpha p+l}{1+l}\right)^{n-i}(r+d)}(z-\omega)^{\alpha p+k}
\end{gathered}
$$

or by

$$
f(z)^{\alpha}=(z-\omega)^{\alpha p}-\frac{\alpha p[\gamma(\beta+2)-1]\left(\frac{1+\lambda(\alpha p-1)+l}{1+l}\right)^{n}}{[1+\gamma \beta+\alpha p(\gamma(\beta+2)-1)]\left(\frac{1+\lambda \alpha p+l}{1+l}\right)^{n}(r+d)}(z-\omega)^{\alpha p+k} .
$$


Corollary 3.2: Let the function $f(z)^{\alpha}$ defined by (4) be in the class $S_{c, n}^{*}(\omega, \alpha, \gamma, \beta, \lambda, p, l)$.

Then, we have

$$
\begin{gathered}
(r+d)^{\alpha p}-\frac{\alpha p(r+d)^{\alpha p}[\gamma(\beta+2)-1]\left(\frac{1+\lambda(\alpha p-1)+l}{1+l}\right)^{n}}{[1+\lambda \beta+\alpha p(\gamma(\beta+2)-1)]\left(\frac{1+\lambda \alpha p+l}{1+l}\right)^{n}} \leq\left|f(z)^{\alpha}\right| \\
\leq(r+d)^{\alpha p}-\frac{\alpha p(r+d)^{\alpha p}[\gamma(\beta+2)-1]\left(\frac{1+\lambda(\alpha p-1)+l}{1+l}\right)^{n}}{[1+\lambda \beta+\alpha p(\gamma(\beta+2)-1)]\left(\frac{1+\lambda \alpha p+l}{1+l}\right)^{n}}
\end{gathered}
$$

for $z \in D$, where $\omega$ is arbitrarily fixed in $D$. The result is best possible for the function $f(z)^{\alpha}$ given by (33).

Theorem 3.3: Let the function $f(z)^{\alpha}$ defined by (4) be in the class

$S_{s c, n-i}^{*}(\omega, \alpha, \gamma, \beta, \lambda, p, l)$.

Then, we have

$$
\begin{gathered}
(r+d)^{\alpha p}\left(\frac{1+\lambda(\alpha p-1)+l}{1+l}\right)^{i}-\frac{\alpha p(r+d)^{\alpha p}\left[\gamma\left(\beta+1-(-1)^{\alpha p}\right)+(-1)^{\alpha p}\right]\left(\frac{1+\lambda(\alpha p-1)+l}{1+l}\right)^{n}}{\left[1+\gamma \beta+\alpha p\left[\gamma\left(\beta+1-(-1)^{\alpha p+1}\right)+(-1)^{\alpha p+1}\right]\right]\left(\frac{1+\lambda \alpha p+l}{1+l}\right)^{n-i}} \\
\leq\left|I_{\omega, p}^{i}(\lambda, l) f(z)^{\alpha}\right| \\
\leq(r+d)^{\alpha p}\left(\frac{1+\lambda(\alpha p-1)+l}{1+l}\right)^{i}+\frac{\alpha p(r+d)^{\alpha p}\left[\gamma\left(\beta+1-(-1)^{\alpha p}\right)+(-1)^{\alpha p}\right]\left(\frac{1+\lambda(\alpha p-1)+l}{1+l}\right)^{n}}{\left[1+\gamma \beta+\alpha p\left[\gamma\left(\beta+1-(-1)^{\alpha p+1}\right)+(-1)^{\alpha p+1}\right]\right]\left(\frac{1+\lambda \alpha p+l}{1+l}\right)^{n-i}}
\end{gathered}
$$

for $z \in D$, where $0 \leq i \leq n$ and $\omega$ is arbitrarily fixed in $D$. This result is sharp. Our next results shall include linear combinations of several functions of the type (4).

\section{4. Convex linear Combination}

Theorem 4.1: Let

$$
f_{\alpha p}(z)^{\alpha}=(z-\omega)^{\alpha p}, \quad \alpha>0, \text { and } p \in N
$$

and

$$
\begin{gathered}
f_{\alpha p+k}(z)^{\alpha} \\
=(z-\omega)^{\alpha p}-\frac{\alpha p\left[\gamma\left(\beta+1-(-1)^{\alpha p}\right)+(-1)^{\alpha p}\right]\left(\frac{1+\lambda(\alpha p-1)+l}{1+l}\right)^{n}}{\left[k(1+\gamma \beta)+\alpha p\left[\gamma\left(\beta+1-(-1)^{\alpha p+k}\right)+(-1)^{\alpha p+k}\right]\right]\left(\frac{1+\lambda(\alpha p+k-1)+l}{1+l}\right)^{n}(r+d)^{k}}(z-\omega)^{\alpha p+k} .
\end{gathered}
$$

Then, $f(z)^{\alpha} \in S_{s, n}^{*}(\omega, \alpha, \gamma, \beta, \lambda, p, l)$ if and only if it can be expressed in the form: 


$$
f(z)^{\alpha}=\sum_{k=0}^{\infty} \delta_{\alpha p+k} f_{\alpha p+k}(z)^{\alpha}
$$

where

$$
\delta_{\alpha p+k} \geq 0 \quad \text { and } \quad \sum_{k=0}^{\infty} \delta_{\alpha p+k}=1
$$

Proof: Let

$$
f(z)^{\alpha}=\sum_{k=0}^{\infty} \delta_{\alpha p+k} f_{\alpha p+k}(z)^{\alpha}=\delta_{\alpha p} f_{\alpha p}(z)^{\alpha}+\sum_{k=1}^{\infty} \delta_{\alpha p+k} f_{\alpha p+k}(z)^{\alpha} .
$$

Using (36), (37), (38) and (39), it is easily seen that

$$
f(z)^{\alpha}
$$

$$
=(z-\omega)^{\alpha p}-\frac{\alpha p\left[\gamma\left(\beta+1-(-1)^{\alpha p}\right)+(-1)^{\alpha p}\right]\left(\frac{1+\lambda(\alpha p-1)+l}{1+l}\right)^{n} \delta_{\alpha p+k}}{\left[k(1+\gamma \beta)+\alpha p\left[\gamma\left(\beta+1-(-1)^{\alpha p+k}\right)+(-1)^{\alpha p+k}\right]\right]\left(\frac{1+\lambda(\alpha p+k-1)+l}{1+l}\right)^{n}(r+d)^{k}}(z-\omega)^{\alpha p+k} .
$$

since

$$
\begin{gathered}
\sum_{k=1}^{\infty} \frac{\left[k(1+\gamma \beta)+\alpha p\left[\gamma\left(\beta+1-(-1)^{\alpha p+k}\right)+(-1)^{\alpha p+k}\right]\right]\left(\frac{1+\lambda(\alpha p+k-1)+l}{1+l}\right)^{n}(r+d)^{k}}{\alpha p\left[\gamma\left(\beta+1-(-1)^{\alpha p}\right)+(-1)^{\alpha p}\right]\left(\frac{1+\lambda(\alpha p-1)+l}{1+l}\right)^{n}} \delta_{\alpha p+k} \\
\bullet \frac{\alpha p\left[\gamma\left(\beta+1-(-1)^{\alpha p}\right)+(-1)^{\alpha p}\right]\left(\frac{1+\lambda(\alpha p-1)+l}{1+l}\right)^{n}}{\left[k(1+\gamma \beta)+\alpha p\left[\gamma\left(\beta+1-(-1)^{\alpha p+k}\right)+(-1)^{\alpha p+k}\right]\right]\left(\frac{1+\lambda(\alpha p+k-1)+l}{1+l}\right)^{n}(r+d)^{k}}=\sum_{k=1}^{\infty} \delta_{\alpha p+k} . \\
1-\delta_{\alpha p} \leq 1 .
\end{gathered}
$$

It follows from theorem 2.1 that the function $f(z)^{\alpha} \in S_{s, n}^{*}(\omega, \alpha, \gamma, \beta, \lambda, p, l)$.

Conversely, let us suppose that $f(z)^{\alpha} \in S_{s, n}^{*}(\omega, \alpha, \gamma, \beta, \lambda, p, l)$. Since

Setting

$$
\begin{gathered}
a_{p+k} \leq \frac{\alpha p\left[\gamma\left(\beta+1-(-1)^{\alpha p}\right)+(-1)^{\alpha p}\right]\left(\frac{1+\lambda(\alpha p-1)+l}{1+l}\right)^{n}}{\left[k(1+\gamma \beta)+\alpha p\left[\gamma\left(\beta+1-(-1)^{\alpha p+k}\right)+(-1)^{\alpha p+k}\right]\right]\left(\frac{1+\lambda(\alpha p+k-1)+l}{1+l}\right)^{n}(r+d)^{k}} \\
k \geq 1, p \in N, n \in N_{0}, \alpha>1,0 \leq \beta \leq 1,0<\gamma \leq 1 \text { and } z \in D .
\end{gathered}
$$

$$
\delta_{\alpha p+k}=\frac{\left[k(1+\gamma \beta)+\alpha p\left[\gamma\left(\beta+1-(-1)^{\alpha p+k}\right)+(-1)^{\alpha p+k}\right]\right]\left(\frac{1+\lambda(\alpha p+k-1)+l}{1+l}\right)^{n}(r+d)^{k}}{\alpha p\left[\gamma\left(\beta+1-(-1)^{\alpha p}\right)+(-1)^{\alpha p}\right]\left(\frac{1+\lambda(\alpha p-1)+l}{1+l}\right)^{n}}
$$

and 


$$
\delta_{\alpha p}=1-\sum_{k=1}^{\infty} \delta_{\alpha p+k}
$$

It follows that $f(z)^{\alpha}=\sum_{k=0}^{\infty} \delta_{\alpha p+k} f_{\alpha p+k}(z)^{\alpha}$ and this completes the proof of the theorem.

Corollary 4.1: The extreme points of the class $S_{s, n}^{*}(\omega, \alpha, \gamma, \beta, \lambda, p, l)$ are functions given by (36) and (37).

Theorem 4.2: Let

$$
f_{\alpha p}(z)^{\alpha}=(z-\omega)^{\alpha p}, \quad \alpha>0, \text { and } p \in N
$$

and

$$
\begin{gathered}
f_{\alpha p+k}(z)^{\alpha}= \\
(z-\omega)^{\alpha p}-\frac{\alpha p[\gamma(\beta+2)-1]\left(\frac{1+\lambda(\alpha p-1)+l}{1+l}\right)^{n}}{\left[1+\gamma \beta+\alpha p\left[\gamma\left(\beta+1-(-1)^{\alpha p+1}\right)+(-1)^{\alpha p+1}\right]\right]\left(\frac{1+\lambda \alpha p+l}{1+l}\right)^{n}(r+d)}(z-\omega)^{\alpha p+k}
\end{gathered}
$$

Then, $f(z)^{\alpha} \in S_{c, n}^{*}(\omega, \alpha, \gamma, \beta, \lambda, p, l)$ if and only if it can be expressed in the form

$$
f(z)^{\alpha}=\sum_{k=0}^{\infty} \delta_{\alpha p+k} f_{\alpha p+k}(z)^{\alpha}
$$

where

$$
\delta_{\alpha p+k} \geq 0 \quad \text { and } \quad \sum_{k=0}^{\infty} \delta_{\alpha p+k}=1 .
$$

The proof is similar to that of theorem 4.1.

Corollary 4.2: The extreme points of the class $S_{c, n}^{*}(\omega, \alpha, \gamma, \beta, \lambda, p, l)$ are functions given by (43) and (44).

Theorem 4.3. Let

$$
\begin{gathered}
f_{\alpha p}(z)^{\alpha}=(z-\omega)^{\alpha p}, \quad \alpha>0, \text { and } p \in N \\
f_{\alpha p+k}(z)^{\alpha}=(z-\omega)^{\alpha p}- \\
\left.\frac{\alpha p\left[\gamma\left(\beta+1-(-1)^{\alpha p}\right)+(-1)^{\alpha p}\right]\left(\frac{1+\lambda(\alpha p-1)+l}{1+l}\right)^{n}}{\left[k(1+\gamma \beta)+\alpha p\left[\gamma\left(\beta+1-(-1)^{\alpha p+k}\right)+(-1)^{\alpha p+k}\right]\right]\left(\frac{1+\lambda(\alpha p+k-1)+l}{1+l}\right)^{n}(r+d)^{k}}(z-\omega)^{\alpha p+k} \cdot 48\right)
\end{gathered}
$$

Then, $f(z)^{\alpha} \in S_{s c, n}^{*}(\omega, \alpha, \gamma, \beta, \lambda, p, l)$ if and only if it can be expressed in the form:

where

$$
f(z)^{\alpha}=\sum_{k=0}^{\infty} \delta_{\alpha p+k} f_{\alpha p+k}(z)^{\alpha}
$$

$$
\delta_{\alpha p+k} \geq 0 \quad \text { and } \quad \sum_{k=0}^{\infty} \delta_{\alpha p+k}=1 .
$$

The proof is also similar to that of theorem 4.1.

Corollary 4.3: The extreme points of the class $S_{s c, n}^{*}(\omega, \alpha, \gamma, \beta, \lambda, p, l)$ are functions given by (47) and (48).

Theorem 4.4: The class $S_{s, n}^{*}(\omega, \alpha, \gamma, \beta, \lambda, p, l)$ is closed under convex linear combination.

Proof: Let us suppose that the function $f_{1}(z)^{\alpha}$ and $f_{2}(z)^{\alpha}$ defined by 


$$
f_{j}(z)^{\alpha}=(z-\omega)^{\alpha p}-\sum_{k=1}^{\infty} a_{p+k, j}(\alpha) \quad(j=1,2 ; z \in)
$$

are in the class $S_{s, n}^{*}(\omega, \alpha, \gamma, \beta, \lambda, p, l)$.

Setting

$$
f(z)^{\alpha}=\mu f_{1}(z)^{\alpha}+(1-\mu) f_{2}(z)^{\alpha}, \quad(0 \leq \mu \leq 1) .
$$

Then from (50), we can write

$$
f(z)^{\alpha}=(z-\omega)^{\alpha p}-\sum_{k=1}^{\infty}\left[\mu a_{p+k, 1}(\alpha)+(1-\mu) a_{p+k, 2}(\alpha)\right](z-\omega)^{\alpha p+k} \quad(0 \leq \mu \leq 1 ; z \in D)(52)
$$

Thus, in view of theorem 2.1, we can have that

$$
\begin{aligned}
\sum_{k=1}^{\infty}[ & \left.k(1+\gamma \beta)+\alpha p\left[\gamma\left(\beta+1-(-1)^{\alpha p+k}\right)+(-1)^{\alpha p+k}\right]\right] \eta(r+d)^{k}\left(\mu a_{p+k, 1}(\alpha)+(1-\mu) a_{p+k, 2}(\alpha)\right) \\
= & \mu \sum_{k=1}^{\infty}\left[k(1+\gamma \beta)+\alpha p\left[\gamma\left(\beta+1-(-1)^{\alpha p+k}\right)+(-1)^{\alpha p+k}\right]\right] \eta(r+d)^{k} a_{p+k, 1}(\alpha) \\
& +(1-\mu) \sum_{k=1}^{\infty}\left[k(1+\gamma \beta)+\alpha p\left[\gamma\left(\beta+1-(-1)^{\alpha p+k}\right)+(-1)^{\alpha p+k}\right]\right] \eta(r+d)^{k} a_{p+k, 2}(\alpha) \\
& \leq \mu \alpha p\left[\gamma\left(\beta+1-(-1)^{\alpha p}\right)+(-1)^{\alpha p}\right] \varsigma+(1-\mu) \alpha p\left[\gamma\left(\beta+1-(-1)^{\alpha p}\right)+(-1)^{\alpha p}\right] \varsigma \\
& =\alpha p\left[\gamma\left(\beta+1-(-1)^{\alpha p}\right)+(-1)^{\alpha p}\right] \varsigma, \\
& \eta=\left(\frac{1+\lambda(\alpha p+k-1)+l}{1+l}\right)^{n} \quad \text { and } \quad \varsigma=\left(\frac{1+\lambda(\alpha p-1)+l}{1+l}\right)^{n}
\end{aligned}
$$

which show that $f(z)^{\alpha} \in S_{s, n}^{*}(\omega, \alpha, \gamma, \beta, \lambda, p, l)$ and this complete the proof of theorem 4.4.

Theorem 4.5: the class $S_{c, n}^{*}(\omega, \alpha, \gamma, \beta, \lambda, p, l)$ is closed under convex linear combination.

Proof: Let us suppose that the function $f_{1}(z)^{\alpha}$ and $f_{2}(z)^{\alpha}$ defined by

$$
f_{j}(z)^{\alpha}=(z-\omega)^{\alpha p}-\sum_{k=1}^{\infty} a_{p+k, j}(\alpha) \quad(j=1,2 ; z \in)
$$

are in the class $S_{c, n}^{*}(\omega, \alpha, \gamma, \beta, \lambda, p, l)$.

Setting

Then from (53), we can write

$$
f(z)^{\alpha}=\mu f_{1}(z)^{\alpha}+(1-\mu) f_{2}(z)^{\alpha}, \quad(0 \leq \mu \leq 1) .
$$

$$
f(z)^{\alpha}=(z-\omega)^{\alpha p}-\sum_{k=1}^{\infty}\left[\mu a_{p+k, 1}(\alpha)+(1-\mu) a_{p+k, 2}(\alpha)\right](z-\omega)^{\alpha p+k}(0 \leq \mu \leq 1 ; z \in D) .
$$

Then from (54), we can write

\section{Radii of starlikeness and convexity}

Theorem 5.1: Let the function $f(z)^{\alpha}$ defined by (4) in the class $S_{s, n}^{*}(\omega, \alpha, \gamma, \beta, \lambda, p, l)$.

Then $f(z)^{\alpha}$ is starlike of order $\varphi(0 \leq \varphi \leq 1)$ in $|z-\omega|<r_{1}$, where

$$
r_{1}=\inf _{k}\left[\frac{(\alpha p-\varphi)\left[k(1+\gamma \beta)+\alpha p\left[\gamma\left(\beta+1-(-1)^{\alpha p+k}\right)+(-1)^{\alpha p+k}\right]\right]\left(\frac{1+\lambda(\alpha p+k-1)+l}{1+l}\right)^{n}(r+d)^{k}}{\alpha p(k-1+\alpha p-\varphi)\left[\gamma\left(\beta+1-(-1)^{\alpha p}\right)+(-1)^{\alpha p}\right]\left(\frac{1+\lambda(\alpha p-1)+l}{1+l}\right)^{n}}\right] \text {. }
$$

The result is best possible with the extremal function given by (15) and $r_{1}$ attains its infimum for $k=1$. 
Proof: It is ample to show that

$$
\left|\frac{(z-\omega)\left(f(z)^{\alpha}\right)^{\prime}}{f(z)^{\alpha}}-\alpha \rho\right|<\alpha \rho-\varphi
$$

for $|z-\omega|<r_{1}$. This implies that

or

$$
\left|\frac{-\sum_{k=1}^{\infty}(k-1) a_{p+k}(\alpha)(z-\omega)^{\alpha p+k}}{(z-\omega)^{\alpha p}-\sum_{k=1}^{\infty} a_{p+k}(\alpha)(z-\omega)^{\alpha p+k}}\right|<\alpha p-\varphi
$$

That is

$$
\frac{\sum_{k=1}^{\infty}(k-1) a_{p+k}(\alpha)|z-\omega|^{k}}{1-\sum_{k=1}^{\infty} a_{p+k}(\alpha)(z-\omega)^{k}}<\alpha p-\varphi .
$$

$$
\frac{\sum_{k=1}^{\infty}(r+d)^{k}\left[k(1+\gamma \beta)+\alpha p\left[\gamma\left(\beta+1-(-1)^{\alpha p+k}\right)+(-1)^{\alpha p+k}\right]\left(\frac{1+\lambda(\alpha p+k-1)+1}{1+l}\right)^{n} a_{p+k}(\alpha)\right.}{\alpha p\left[\gamma\left(\beta+1-(-1)^{\alpha p}\right)+(-1)^{\alpha p}\right]\left(\frac{1+\lambda(\alpha p+k-1)+1}{1+l}\right)^{n}} \leq
$$$$
\frac{\sum_{k=1}^{\infty}(k-1+\alpha p-\varphi) a_{p+k}(\alpha)|z-\omega|^{k}}{\alpha p-\varphi} \leq 1 .
$$

Hence, (57) is proven true if

$$
\leq \frac{\frac{(k-1+\alpha p-\varphi)|z-\omega|^{k}}{\alpha p-\varphi}}{(r+d)^{k}\left[k(1+\gamma \beta)+\alpha p\left[\gamma\left(\beta+1-(-1)^{\alpha p+k}\right)+(-1)\right]\left[\left(\frac{1+\lambda(\alpha p+k-1)+1}{1+l}\right)^{n}\right.\right.} .
$$

That is,$$
|z-\omega| \leq\left[\frac{(\alpha p-\varphi)\left[k(1+\gamma \beta)+\alpha p\left[\gamma\left(\beta+1-(-1)^{\alpha p+k}\right)+(-1)^{\alpha p+k}\right]\right]\left(\frac{1+\lambda(\alpha p+k-1)+l}{1+l}\right)^{n}(r+d)^{k}}{\alpha p(k-1+\alpha p-\varphi)\left[\gamma\left(\beta+1-(-1)^{\alpha p}\right)+(-1)^{\alpha p}\right]\left(\frac{1+\lambda(\alpha p-1)+l}{1+l}\right)^{n}}\right]^{\frac{1}{k}} k \geq 1
$$

and this ends the proof of theorem 5.1.

Remark: It is clear that $r_{1}$ attains its infimum at $k=1$ for the function $f(z)$ given by

$$
f(z)^{\alpha}=(z-\omega)^{\alpha p}-\frac{\alpha p\left[\gamma\left(\beta+1-(-1)^{\alpha p}\right)+(-1)^{\alpha p}\right]\left(\frac{1+\lambda(\alpha p-1)+l}{1+l}\right)^{n}}{\left[1+\gamma \beta+\alpha p\left[\gamma\left(\beta+1-(-1)^{\alpha p+1}\right)+(-1)^{\alpha p+1}\right]\left[\frac{1+\lambda \alpha p+l}{1+l}\right)^{n}\right.}(z-\omega)^{\alpha p+k} .
$$

Theorem 5.2: Let the function $f(z)^{\alpha}$ defined by (4) be in the class $S_{c, n}^{*}(\omega, \alpha, \gamma, \beta, \lambda, p, l)$. Then $f(z)^{\alpha}$ is starlike of order $\varphi(0 \leq \varphi \leq 1)$ in $|z-\omega|<r_{2}$, where 


$$
r_{2}=\inf _{k}\left[\frac{(\alpha p-\varphi)[k(1+\gamma \beta)+\alpha p[\gamma(\beta+2)-1]]\left(\frac{1+\lambda(\alpha p+k-1)+1}{1+l}\right)^{n}(r+d)^{k}}{\alpha p[\gamma(\beta+2)-1]\left(\frac{1+\lambda(\alpha p+k-1)+1}{1+l}\right)^{n}}\right] .
$$

The result is best possible with the extremal function given by (18) and $r_{2}$ attains its infimum for $k=1$. The proof is similar to that of Theorem 5.1.

Theorem 5.3: Let the function $f(z)^{\alpha}$ defined by (4) be in the class $S_{s c, n}^{*}(\omega, \alpha, \gamma, \beta, \lambda, p, l)$. Then $f(z)^{\alpha}$ is starlike order $\varphi(0 \leq \varphi \leq 1)$ in $|z-\omega|<r_{3}$, where

$$
r_{3}=\inf _{k}\left[\frac{(\alpha p-\varphi)\left[k(1+\gamma \beta)+\alpha p\left[\gamma\left(\beta+1-(-1)^{\alpha p+k}\right)+(-1)^{\alpha p+k}\right]\left(\frac{1+\lambda(\alpha p+k-1)+1}{1+l}\right)^{n}(r+d)^{k}\right.}{(k+\alpha p)(k+\alpha p-\varphi)\left[\gamma\left(\beta+1-(-1)^{\alpha p}\right)+(-1)^{\alpha p}\right]\left(\frac{1+\lambda(\alpha p-1)+1}{1+l}\right)^{\frac{1}{k+}}}\right]
$$

Proof: It is ample to show that

$$
\left|1+\frac{(z-\omega)\left(f(\beta)^{\alpha}\right)^{\prime \prime}}{\left(f(\beta)^{\alpha}\right)^{i}}-\alpha p\right| \leq \alpha p-\varphi
$$

for $|z-\omega|<r_{1}$. This implies that

$$
\left|\frac{-\sum_{k=1}^{\infty} k(\alpha p+k) a_{p+k}(\alpha)(z-\omega)^{\alpha p+k-1}}{\alpha p(z-\omega)^{\alpha p-1}-\sum_{k=1}^{\infty}(\alpha p-k) a_{p+k}(\alpha)(z-\omega)^{\alpha p+k-1}}\right|<\alpha p-\varphi
$$

or

$$
\left|\frac{-\sum_{k=1}^{\infty} \frac{k(\alpha p+k)}{\alpha p} a_{p+k}(\alpha)(z-\omega)^{k}}{1-\sum_{k=1}^{\infty} \frac{(\alpha p+k)}{\alpha p} a_{p+k}(\alpha)(z-\omega)^{k}}\right|<\alpha p-\varphi .
$$

That is,

$$
\frac{\sum_{k=1}^{\infty}(k+\alpha p)(k+\alpha p-\varphi) a_{p+k}(\alpha) z-\left.\omega\right|^{k}}{\alpha p(\alpha p-\varphi)} \leq 1 .
$$

Now, (61) is proven true if

$$
\begin{gathered}
\frac{(k+\alpha p)(k+\alpha p-\varphi) a_{p+k}(\alpha) z-\left.\omega\right|^{k}}{\alpha p(\alpha p-\varphi)} \\
\leq \frac{(r+d)^{k}\left[k(1-\gamma \beta)+\alpha p\left[\gamma\left(\beta+1-(-1)^{\alpha p+k}\right)+(-1)^{\alpha p+k}\right]\left(\frac{1+\lambda(\alpha p+k-1)+1}{1+l}\right)^{n}\right.}{\alpha p\left[\gamma\left(\beta+1-(-1)^{\alpha p}\right)+(-1)^{\alpha p}\right]\left(\frac{1+\lambda(\alpha p-1)+1}{1+l}\right)^{n}} .
\end{gathered}
$$

Solving for $|z-\omega|$, we have that 


$$
|z-\omega|=\left|\frac{(\alpha p-\varphi)\left[k(1-\gamma \beta)+\alpha p\left[\gamma\left(\beta+1-(-1)^{\alpha p+k}\right)+(-1)^{\alpha p+k}\right]\left[\left(\frac{1+\lambda(\alpha p+k-1)+1}{1+l}\right)^{n}(r+d)^{k}\right.\right.}{(k+\alpha p)(k+\alpha p-\varphi)\left[\gamma\left(\beta+1-(-1)^{\alpha p}\right)+(-1)^{\alpha p}\right]\left(\frac{1+\lambda(\alpha p-1)+1}{1+l}\right)^{\frac{1}{k}}}\right| .
$$

This completes the proof of theorem 5.3.

\section{References}

[1]. M. Acu, S. Owa. On some subclass of univalent functions, J. Inequalitity in Pure and Appl. Math., 6 (3) $70(2005), 1-14$.

[2]. M. K. Aouf, R.M. El-Ashwali, S.M. El-Deeb, Certain classes of Univalent functions with negative coefficient and n-starlike with respect to certain points, Malemankn Bectink, 62(3) (2010), 215-226.

[3]. M.K Aouf, A.Shamandy, A.O Mostafa, and S.M Madian, A subclass of $m-\omega-$ starlike functions, Acta Universitation Apulensis No 21 (2010), 135-142.

[4]. J. Dziok, On the convex combination of the Dziok-Srivastava operator, Appl. Math. Comput., 188(2006), 1214-1220.

[5]. R.M. El-Ashwah, D.K. Thomas, Some subclass of close-to-convex functions, J. Ramaunjan. Math. Soc., 2(1987), 86-100

[6]. S.A. Halim, A. Janteng, M. Darus, Coefficient properties for classes with negative coefficient and starlike with respect to other points, proceeding of the $13^{\text {th }}$ Math. Sci. Nat. Symposium, UEM., 2(2005), 658-663.

[7]. S.Kanas, F. Ronning, Uniformly starlike and Convex functions and related classes of univalent functions, Ann. Univ. Mariae Curie - Sklodowska Section A, 53 (1999), 95-105.

[8]. A.T. Oladipo, On subclasses of analytic and univalent functions, Advances in Applied Mathematical Analysis functions, 4 (1) (2009), 87-93. 